ARTÍCULOS 



\title{
ESTIMACIÓN DE LA VELOCIDAD DEL FLUJO DEL AGUA EN CAUCES EFIMEROS NO AFORADOS A PARTIR DE DATOS LIDAR Y GPS-RTK
}

\author{
Gerson R. Colmenárez López \\ Departamento de Tecnología Agropecuaria, Cátedra Topografía y Dibujo. Universidad Centroccidental 'Lisandro Alvarado'. \\ Barquisimeto.Venezuela \\ Francesca Segura Beltrán \\ Departament de Geografia. Universitat de València \\ Josep E. Pardo Pascual, Luis A. Ruiz Fernández y Jesús Palomar Vázquez \\ Grup Cartografia Geo-Ambiental i Teledetecció, Dept. d'Enginyeria Cartogràfica, Geodèsia i Fotogrametria. \\ Universitat Politècnica de València \\ jepardo@cgf.upv.es
}

\section{RESUMEN}

La fórmula de Manning es una de las más usadas para calcular la velocidad media del flujo del agua en cauces no aforados. Para aplicarla es necesario medir en campo el radio hidráulico $(\mathrm{RH})$, la pendiente de la lámina de agua (S) y obtener el coeficiente de rugosidad (n) generalmente a través de tablas creadas para este fin. Esto implica una ardua, poco eficiente y subjetiva toma de datos en campo. En este estudio se evalúa la posibilidad de obtener dichos parámetros de forma automatizada, lo que disminuye la subjetividad a la vez que ahorra tiempo al permitir trabajar sobre espacios más amplios. Para lograr estos objetivos, en este trabajo se han utilizado: 1) modelos digitales del terreno generados a partir de datos LiDAR, con una densidad de dos puntos por metro cuadrado y que fueron obtenidos cuando el cauce estaba seco; 2) las cotas de la lámina de agua y el perímetro de mojado del cauce medidas en el campo mediante GPS cuando pasaba agua; 3) medidas de caudal cuando se registró circulación. Con esta información se estimó la velocidad (Ve), que se relacionó con la velocidad media del flujo del agua ( $\mathrm{Vc}$ ), calculada a partir de datos medidos en campo. El coeficiente de determinación entre las dos velocidades fue de $73,52 \%$, lo cual sugiere que la metodología propuesta es útil para obtener la velocidad media del flujo, especialmente en zonas de difícil acceso o en cauces secos.

Fecha de recepción: julio 2011.

Fecha de aceptación: enero 2013. 
Palabras clave: Modelo de rugosidad topográfica, tecnología LiDAR, coeficiente n-Manning, modelo digital de elevaciones, velocidad media del agua, cauces naturales, caudal, GPS-RTK y texturas.

\section{ABSTRACT}

The Manning formula is one of the most used to calculate the average velocity of water flow in ungauged channels. In order to be applied, it is necessary to measure in field the hydraulic radius $(\mathrm{RH})$, the slope of the water surface $(\mathrm{S})$, and to obtain the roughness coefficient (n), usually through tables created for this purpose. This involves a difficult, inefficient and subjective data collection in the field. This study evaluates the possibility to obtain these parameters in a more efficient manner, reducing the time of the process and allowing to work in larger areas. To achieve these objectives, the data used in this work were: 1) digital terrain models generated from airborne LiDAR data with a density of 2 points/ $\mathrm{m}^{2}$, acquired when the channel was dry; 2) the height of the waterline and channel geometry measurements in the field using GPS; 3) Flow measurements. With this information, the velocity (Ve) was estimated and related to the average velocity of water flow ( $\mathrm{Vc}$ ) calculated from data measured in the field. The coefficient of determination between both velocity values was $73.52 \%$, suggesting that the proposed methodology is useful to obtain the average velocity of flow, especially in remote areas or dry riverbeds.

Key words: topographic roughness model, LiDAR, n-Manning coefficient, digital elevation model, average water velocity, natural streams, flow, GPS-RTK, texture variables.

\section{INTRODUCCIÓN}

En los últimos años hemos asistido a una auténtica eclosión de estudios donde se evalúa el potencial que presenta la tecnología LiDAR como una nueva fuente de información geográfica para realizar estudios geomorfológicos e hidrológicos. Así por ejemplo, Mouton (2005) generó una metodología para crear mapas de líneas de drenaje a partir de modelos digitales del terreno (MDT) derivados de datos LiDAR y con un Modelo Digital de Elevaciones (MDE) de $10 \mathrm{~m}$ de tamaño de celda. Autores como Glenn et al., (2006), McKean y Roering (2004) y Staley et al., (2006) utilizaron datos LiDAR para caracterizar y diferenciar la morfología y actividad de los movimientos de masa. Análogamente, Frankel et al., (2007) lograron determinar satisfactoriamente el desplazamiento de dos abanicos aluviales gracias a la precisión y exactitud que ofrece la tecnología LiDAR. Más recientemente, autores como Cavalli et al., (2008) han utilizado MDE de muy alta resolución con el objetivo de reconocer mesoformas en los cauces fluviales. Gilveart et al. (2004), usando imágenes multiespectrales, fotografías aéreas en color y datos LiDAR lograron caracterizar de manera satisfactoria la morfología de un tramo de cauce $(8 \mathrm{~km})$ del río Tummel en Escocia, alcanzando una precisión superior al $99 \%$ al clasificar de manera automatizada islas, rocas expuestas y láminas de agua, si bien al tratar de establecer una clasificación más precisa (rápidos, remolinos y remansos) la precisión alcanzada fue inferior al 70\%. Recientemente, Colmenárez et al. 
(2010) calcularon la $n$ de Manning de manera indirecta a partir de un modelo digital de rugosidades (MDR) generado a partir de un modelo digital de elevaciones creado a partir de datos LiDAR (MDE-LiDAR) de alta resolución. Por su parte, Casas et al. (2010), en un sector del llano de inundación del Ter, exploran el papel de la complejidad topográfica en la parametrización de la modelización hidráulica, teniendo en cuenta la dependencia entre la rugosidad y la topografía. Mediante el uso de modelos 2D calculan la rugosidad usando una ecuación basada en la teoría de la lámina de mezcla (Katul et al., 2002).

La medición de la velocidad en ríos no aforados o de difícil acceso es bastante complicada. Para solventar estas dificultades, en el presente trabajo nos planteamos calcular la velocidad del flujo del agua de forma semi-automatizada a partir de datos LiDAR aerotransportado y medidas GPS realizadas en campo, contrastando su nivel de calidad con datos de velocidad medidos en el cauce.

De acuerdo con Knighton (1998) la velocidad es uno de los parámetros más variables en un cauce, ya que depende de una gran cantidad de factores. En ríos no aforados, la fórmula de Manning [1] se ha usado tradicionalmente para calcular la velocidad media del flujo (Giogia y Bombardelli, 2002), dada su sencillez de aplicación. Dicha fórmula obtiene la velocidad a partir de la pendiente de la lámina de agua $(S)$, el radio hidráulico $(R h)$, y el coeficiente de rugosidad $n$.

$$
V=\left[R h^{2 / 3} \cdot S^{1 / 2}\right] / n[1] .
$$

El empleo de datos topográficos de detalle, puede resultar insuficiente para caracterizar la rugosidad real a la que está sometido un flujo. Este parámetro expresa la rugosidad total del cauce producida por el calibre de los sedimentos, la forma de la sección transversal, la presencia de vegetación e incluso a la fricción de la superficie del agua con el aire (Robert, 2003), aunque otros autores (Cowan, 1956; Chow, 2000) incluyen parámetros como el índice de meandrización y el grado de flexibilidad y porte de la vegetación. Para obviar el último factor, en este caso se han seleccionado parcelas libres de vegetación.

\section{II. ÁREA DE ESTUDIO}

El presente estudio se ha desarrollado en el cauce medio del río Palancia, $\left(976 \mathrm{~km}^{2}\right.$ de cuenca, unos $90 \mathrm{~km}$ de longitud y $1,5 \%$ de pendiente media) que drena la parte meridional del Sistema Ibérico desembocando en el mar en la población del Port de Sagunt (Valencia). La litología dominante de la cuenca son las areniscas silíceas triásicas con algunos afloramientos de materiales calcáreos. Se trata de un río mediterráneo, con caudal casi permanente en su cuenca alta, pero efímero en la parte baja. En cabecera, su caudal medio es de $1,5 \mathrm{~m}^{3} / \mathrm{s}$ pero aguas abajo sus aguas son retenidas por dos presas (presa del Regajo y presa de l'Algar). Debido a la existencia de dichos embalses y a las pérdidas por transmisión, la cuenca baja solo registra caudal después de precipitaciones muy intensas.

El área de estudio se encuentra entre las poblaciones del Algar de Palancia y Torres Torres, (Fig. 1). En este sector la morfología del cauce es trenzado siendo abundantes las barras e islas fluviales formadas principalmente por sedimentos de calibre grueso (cantos y bloques). Dentro de este sector se han seleccionado cinco zonas de estudio en las que se ubicaron 43 parcelas, cuya distribución se puede apreciar en la tabla 1. 
Para seleccionar las parcelas de estudio (tabla 1) se eligieron tramos fluviales de trazado y morfología uniforme, exentos de alteraciones antrópicas y sin confluencias intermedias. La ausencia de vegetación fue un factor determinante. Las 43 parcelas objeto de estudio tenían una longitud promedio de 17,87 m con una desviación estándar de 6,99 m, siendo la mayor longitud de $40,46 \mathrm{~m}$ y la menor de $5,38 \mathrm{~m}$.

El caudal medio estimado a partir de las medidas de campo durante los quince días en que se realizó el trabajo osciló entre $0,089 \mathrm{~m}^{3} / \mathrm{s}(24 / 02 / 09)$ y $2,95 \mathrm{~m}^{3} / \mathrm{s}(2 / 11 / 08)$, fecha en la que se registró una pequeña crecida debido a las lluvias caídas.

Tabla 1

COORDENADAS UTM (HUSO 30) EN EL SISTEMA WGS-84 DE LAS CINCO ZONAS DE ESTUDIO Y PARCELAS DE ESTUDIO QUE LAS CONFORMAN

\begin{tabular}{|c|c|c|c|c|c|}
\hline \multirow{2}{*}{$\begin{array}{c}\text { Zona de } \\
\text { Estudio }\end{array}$} & \multicolumn{2}{|c|}{$\begin{array}{c}\text { Coord. Esquina superior } \\
\text { Izquierda }\end{array}$} & \multicolumn{2}{c|}{$\begin{array}{c}\text { Coord. Esquina inferior } \\
\text { Derecha }\end{array}$} & \multirow{2}{*}{ Parcelas de Estudio } \\
\cline { 2 - 6 } & $\mathbf{X}$ & $\mathbf{Y}$ & $\mathbf{X}$ & $\mathbf{Y}$ & \\
\hline 1 & 725.529 & 4.406 .989 & 726.295 & 4.406 .173 & De la 1 a la 8 \\
\hline 2 & 726.648 & 4.405 .125 & 727.027 & 4.404 .445 & De la 9 a la 12 \\
\hline 3 & 727.174 & 4.403 .388 & 728.341 & 4.402 .630 & De la 13 a la 25 \\
\hline 4 & 728.426 & 4.402 .256 & 728.791 & 4.402 .061 & De la 26 a la 37 \\
\hline 5 & 728.587 & 4.400 .807 & 728.838 & 4.400 .289 & De la 38 a la 43 \\
\hline
\end{tabular}

Figura 1

UBICACIÓN RELATIVA DE LAS 5 ÁREAS DE ESTUDIO Y FOTOGRAFIAS DE PARCELAS DE MUESTRA

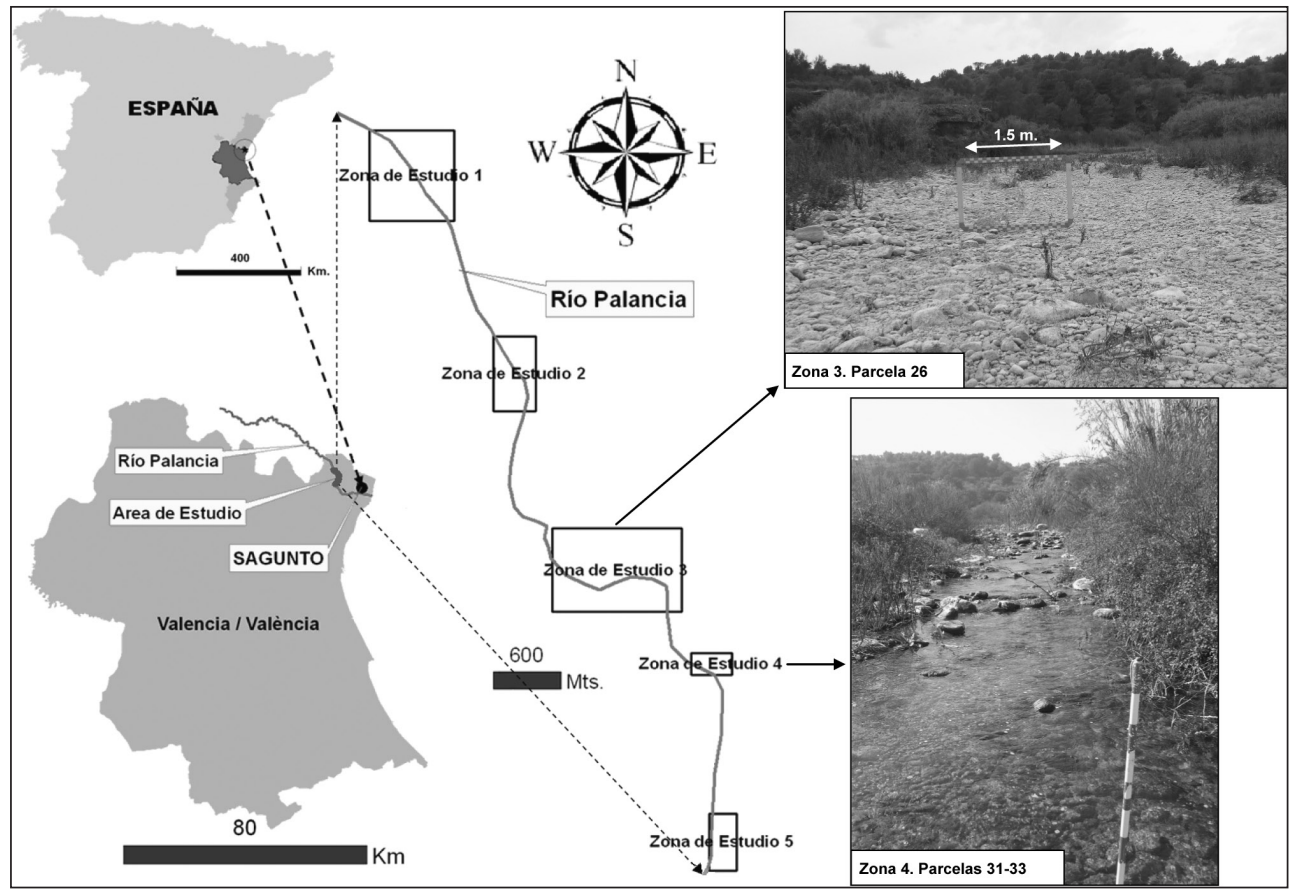




\section{METODOLOGÍA}

Para conseguir los objetivos que se han propuesto, se ha desarrollado una metodología que requiere la siguiente información:

$\checkmark$ Datos LiDAR del cauce seco. En este caso se ha trabajado con los datos procedentes de un levantamiento LiDAR realizado el 24 agosto de 2009.

$\checkmark$ Medidas en el cauce de la cota y la geometría del cauce con GPS-RTK.

$\checkmark$ Medidas realizadas en campo de los datos necesarios para calcular la velocidad media del flujo (Vc) cuando el cauce llevaba agua. En este caso las mediciones se realizaron en noviembre del 2008, febrero y marzo del 2009 y mayo del 2010. En estas fechas el río llevaba flujo debido a pequeños aportes locales.

\section{Mediciones del caudal realizadas en campo}

Con la finalidad de obtener los datos hidráulicos necesarios para el cálculo de velocidades de campo $(\mathrm{Vc})$ y la pendiente de la lámina de agua $(S)$ para cada una de las 43 parcelas que conforman este estudio, se midieron las secciones transversales, la velocidad, las cotas de la lámina de agua y la profundidad del flujo en las parcelas estudiadas. Las cotas de la lámina de agua se han usado para calcular la pendiente de dicha lámina a partir de la fórmula:

$$
S=\left(C_{a}-C_{b}\right) / D H_{a b}[2]
$$

donde:

$S$ : Pendiente de la lámina de agua en $\mathrm{m} / \mathrm{m}$.

$C_{a}$ : cota inicial de la lámina de agua, la medida aguas arriba (punto a).

$C_{b}$ : cota final de la lámina, medida aguas abajo (punto b).

$\mathrm{DH}_{a b}$ : distancia horizontal medida entre los puntos a y b.

Figura 2

MEDICIONES REALIZADAS EN LAS PARCELAS DE ESTUDIO (TRAMO DEL RIOO)

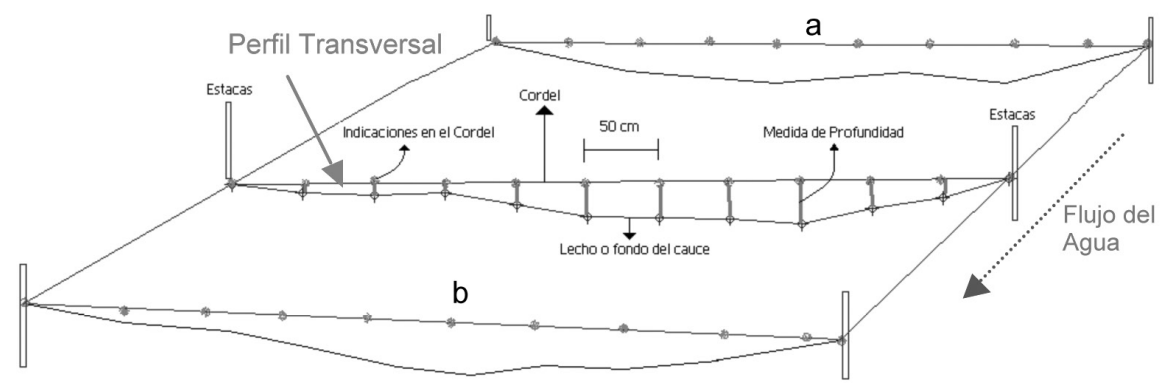

Estas mediciones se realizaron con el equipo GPS-RTK (Leica Smarth Rover), con correcciones diferenciales en tiempo real vía Internet y con una precisión mejor que $3 \mathrm{~cm}$ tanto en planimetría como en altimetría (Lou y Chen, 2008). El radio hidráulico se ha obtenido a partir de los perfiles transversales (Fig. 3) generados automáticamente (RHMed) a partir del software diseñado para tal fin (ver apartado 4.3). 


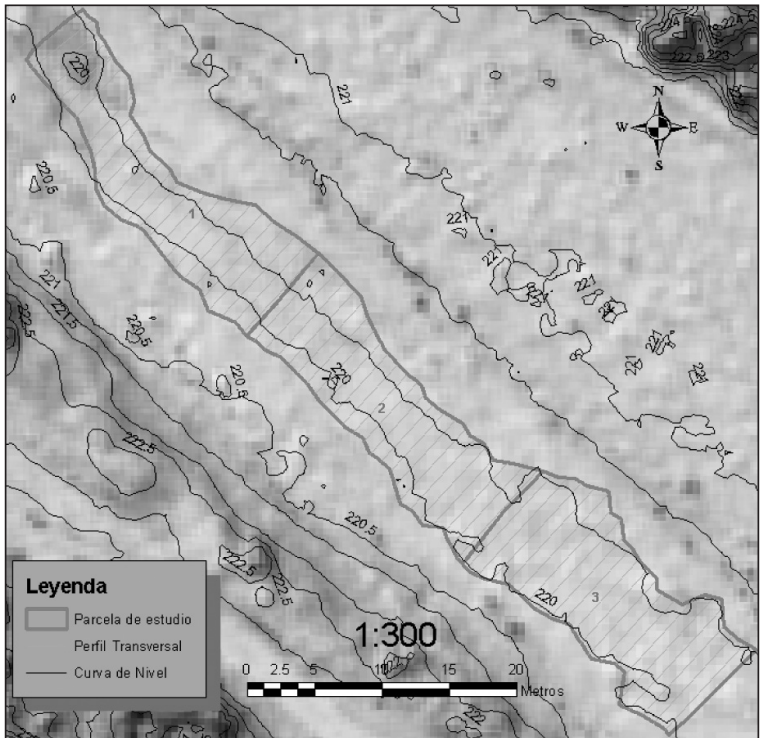

La velocidad media del agua se midió usando flotadores esféricos de $40 \mathrm{~mm}$ de diámetro, (Whiting, 2003). En cada parcela de estudio se cronometró el tiempo empleado por la esfera en desplazarse desde el punto «a» al punto «b» (Fig. 2) al menos cuatro veces, obteniéndose así un tiempo promedio, que no debe ser inferior a 20 segundos (Whiting, 2003). En este estudio, el tiempo medio medido para todas las parcelas analizadas fue de 45 segundos. Conocida la distancia entre los puntos «a» $\mathrm{y}$ «b» se calculó la velocidad media del flujo del agua para las 43 parcelas a partir de la ecuación [3].

$$
V_{a b}=D H_{a b} / T_{a b}
$$

donde:

$V_{a b}=$ Velocidad promedio en la parcela.

$D H a b=$ Distancia que separa el punto «a» del punto «b».

$T a b=$ Tiempo promedio que tarda el flotador en recorrer la distancia de $\langle\mathrm{a} »$ hasta $« \mathrm{~b} »$.

\section{Deducción del coeficiente $n$ de Manning a partir de datos LiDAR}

El valor del coeficiente $n$ de Manning se ha obtenido tradicionalmente a partir de tablas diseñadas para tal fin (Chow et al., 1994), lo cual introduce un alto grado de subjetividad. En nuestro caso se ha utilizado la metodología propuesta por Colmenárez et al., (2010), que estima el valor del coeficiente $n$ de la fórmula de Manning a partir de un modelo digital del terreno que cuantifica la rugosidad de la superficie del cauce y que ha sido generado a partir de datos LiDAR y GPS. Los datos LiDAR se obtuvieron mediante un sistema LiDAR Optech ALTM 3025 con una densidad nominal de 2 puntos $/ \mathrm{m}^{2}$ y una densidad media final 
de 3,1 puntos $/ \mathrm{m}^{2}$ el 24 de agosto de 2009, cuando el cauce del río estaba seco. El MDE se generó mediante el programa FUSION (McGaughey, 2008), obteniendo en primer lugar el conjunto de puntos que pertenecen al suelo mediante un algoritmo de filtrado (adaptado de Kraus y Pfeifer, 1998) basado en un procedimiento iterativo de generación de superficies situadas entre el suelo y la parte superior de la vegetación, de forma que en cada iteración se calculan las distancias y orientaciones de los puntos con respecto a las superficies, modificando los parámetros y recalculando la superficie hasta que se optimiza su ajuste a los puntos del terreno. Posteriormente, se genera el MDE en formato raster asignando a cada píxel el valor medio de los puntos situados dentro del mismo. En nuestro caso, la resolución espacial del MDE obtenido es de $1 \mathrm{~m} /$ píxel. Una vez calculado el MDE, el procedimiento seguido ha sido el siguiente:

a) se ha calculado el coeficiente $n$ de Manning a partir de los datos medidos en campo (n_Campo) y el radio hidráulico promedio (RHMed) generado automáticamente a partir del software diseñado para tal fin (ver apartado 4.3), promediando al menos 5 perfiles transversales por parcela de estudio (Fig. 3);

b) se han generado 5 variables provenientes del estudio de la matriz de ocurrencias obtenidas del análisis de textura (intervalo de valores, media, varianza, entropía y asimetría) más una generada a partir de las mediciones del vector gradiente (según la metodología propuesta por Álvarez et al., 2002);

c) el coeficiente $n$ de Manning «n_Deducida» (n_D) se calculó a partir de un análisis de correlación múltiple en el que usaron como variables independientes las seis antes mencionadas, y como variable dependiente la n_Campo. Para tal fin se usó el software StatgraphicsPlus (C). El método de regresión múltiple usado fue el de paso a paso hacia atrás con una $F$-to-Enter y $F$-to-Remove de 4 ; al final de este análisis estadístico quedó la variable varianza $(V)$ proveniente de la matriz de ocurrencias del análisis de texturas como la de mayor influencia en el modelo. La relación lineal entre la n_Campo versus la Varianza $(V)$, mejora los resultados obtenidos por Colmenárez et al., (2010) ya que el coeficiente de determinación $\mathrm{R}^{2}$ pasó del $50 \%$ al $55,81 \%$. Sin embargo, el mejor ajuste, se obtiene con una relación polinómica de $2^{\circ}$ orden (tabla 2).

\section{Tabla 2}

ESTADÍSTICOS QUE PRESENTA LA RELACIÓN ENTRE LA N DEDUCIDAA PARTIR DE LOS DATOS DE CAMPO CON LA VARIANZA PROVENIENTE DEL ANÁLISIS DE TEXTURAS

\begin{tabular}{|c|c|c|c|c|}
\hline Modelo & P-Value & $\mathbf{R}^{\mathbf{2}}(\boldsymbol{\%})$ & EMA & Ecuación \\
\hline Lineal & $<0,01$ & 55,81 & 0,0115 & $n \_D=0,0400842+0,920775 * V[4]$ \\
\hline Polinómico $2^{\circ}$ & $<0,01$ & 56,51 & 0,0112 & $n \_D=-3,34274^{*} V^{\wedge} 2+1,20899 * V+0,0384377[5]$ \\
\hline
\end{tabular}

Hay que resaltar que en este estudio, la mayoría de tramos analizados son poco rugosos (valores de $n<0,060$ ), aunque en 11 parcelas analizadas el coeficiente $n$ de Manning está por encima de dicho valor, lo que introduce una mejor representatividad de la variabilidad real de los cauces y da mayor robustez a los modelos estadísticos generados [4] y [5]. Los resultados sugieren que debería usarse el valor de la n de Manning deducida por el modelo presentado en la ecuación [5] con el ajuste polinómico de $2^{\circ}$ para el resto de los cálculos. 


\section{Herramienta de extracción automática de los parámetros morfométricos}

Para realizar las pruebas ha sido necesaria la programación de una herramienta que ofrezca una manera rápida y eficiente de crear y analizar los parámetros morfométricos del área del cauce en base a la creación automática de perfiles transversales perpendiculares al eje central del cauce. Dicha herramienta se ha realizado utilizando ArcMap (C) como entorno de visualización y programación. Los datos de partida para el análisis son los siguientes: el modelo digital de elevaciones generado a partir de los datos LiDAR (MDE-LiDAR) en formato raster, el cauce de estudio y el eje del cauce que se deduce de forma automática a partir de datos del MDE, ambos en formato vectorial. La herramienta consta de tres módulos:

a) El primer módulo permite generalizar el eje central del cauce (Fig. 4a). Dado que en muchas ocasiones, este eje presenta una elevada sinuosidad (Fig. 4b), con esta herramienta se consigue que los perfiles generados se dispongan de forma totalmente perpendicular al cauce real. El proceso de generalización se ha desarrollado aplicando dos tipos de algoritmos encadenados: algoritmos de eliminación de puntos de Douglas-Peucker (1973) y de suavizado polinomial o método PAEK (Bodansky, et al, 2002).

b) El segundo módulo (Fig. 3) crea los perfiles transversales para lo que hay que indicar la distancia entre perfiles, el ancho de la sección transversal y la separación a la que se tomarán datos de cota para construir cada perfil transversal. Para este estudio los valores usados para estos tres parámetros fueron: $1 \mathrm{~m}, 30 \mathrm{~m}$ y $0,5 \mathrm{~m}$ respectivamente. El resultado final es el fichero de perfiles transversales ajustados al ancho del cauce y que contiene la información relevante para calcular la pendiente y el radio hidráulico promedio para cada parcela.

c) Finalmente el tercer módulo (Fig. 5), permite determinar a voluntad la longitud de las parcelas de estudio sobre el polígono que represente al cauce. De esta forma se puede obtener una representación más detallada de las secciones transversales en caso de que se necesite una mayor resolución. Teniendo en cuenta la longitud de estudio, la herramienta genera una tabla resumen en la que se consideran todos los perfiles incluidos dentro de dicha longitud.

(a)

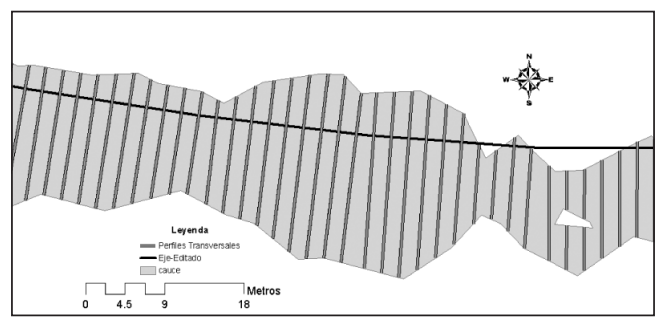

(b)

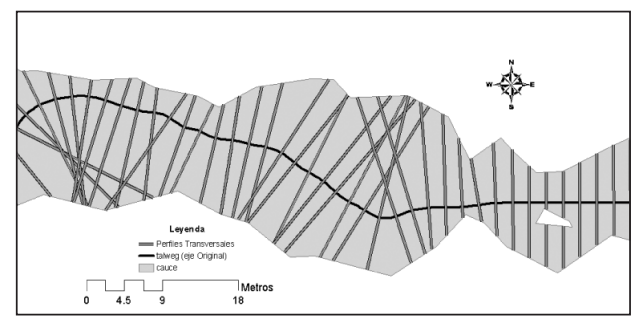


Dicha tabla (Fig. 5), contiene todos los datos resumidos para los perfiles implicados, así como los estadísticos básicos para el radio hidráulico (medio, máximo, mínimo, rango y desviación estándar) y la pendiente (pendiente media y pendientes tanto en el margen derecho como en el margen izquierdo del cauce). Por otro lado y con el objeto de identificar y gestionar posteriormente las parcelas y los perfiles que forman parte de ellas, se han creado campos que representan el identificador de parcela y los identificadores del primer y último perfil que conforman cada parcela.

Figura 5

EXTRACTO DE LA TABLA RESUMEN OBTENIDA CON EL MÓDULO 3

\begin{tabular}{|r|r|r|r|r|r|r|r|r|r|r|}
\hline Parcela & \multicolumn{1}{|c|}{ Perini } & \multicolumn{1}{|c|}{ PerFin } & PenFP & \multicolumn{1}{|c|}{ PenTP } & PenMed & RHMed & RHMax & RHMin & RHRango & RHDesv \\
\hline 192 & 0 & 192 & -1.868722 & -2.070373 & -1.969547 & 0.355973 & 1.282832 & 0.016751 & 1.266082 & 0.232778 \\
\hline 332 & 192 & 332 & -0.720837 & -0.633896 & -0.677366 & 0.227346 & 0.624473 & 0.051833 & 0.57264 & 0.108586 \\
\hline 483 & 332 & 483 & -1.436947 & -1.39424 & -1.415593 & 0.433483 & 1.289367 & 0.020581 & 1.268786 & 0.241326 \\
\hline 643 & 483 & 643 & -2.511498 & -1.89567 & -2.203584 & 0.432437 & 1.37558 & 0.016577 & 1.359004 & 0.28954 \\
\hline 804 & 643 & 804 & -1.694257 & -2.293969 & -1.994113 & 0.420113 & 1.566169 & 0.008637 & 1.557532 & 0.287562 \\
\hline 948 & 804 & 948 & -2.102832 & -1.992143 & -2.047488 & 0.358992 & 1.862015 & 0.003261 & 1.858754 & 0.328921 \\
\hline 1100 & 948 & 1100 & -1.952627 & -2.053853 & -2.00324 & 0.287402 & 0.993264 & 0.009919 & 0.983345 & 0.154683 \\
\hline 1269 & 1100 & 1269 & -1.603892 & -1.80693 & -1.705411 & 0.407477 & 1.128787 & 0.000048 & 1.128739 & 0.231255 \\
\hline
\end{tabular}

Con la información generada, se procede a calcular la velocidad del flujo del agua estimada (Ve) a partir de la fórmula empírica de Manning [1], usando el radio hidráulico medio (RHMed) obtenido automáticamente a partir del Software, la pendiente de la línea de agua medida en campo con GPS $(S$ ) y el coeficiente n de Manning deducido (n_D) a partir del análisis de correlación múltiple y del modelo que describe la rugosidad topográfica generado a partir del «MDE-LiDAR».

\section{Creación de una primera aproximación de un mapa de velocidades}

Para crear el mapa de velocidades (Fig. 7) se usará la fórmula propuesta por ManningLimerinos [6], para lo cual se calculará la profundidad $(d)$ en cada píxel de la parcela de estudio, la pendiente $S$ calculada a partir de los datos GPS y la ecuación [2] y se aplicará la ecuación [5] al modelo de Varianza para deducir en cada píxel el coeficiente $n$ de la fórmula de Manning. Con todo ello se calcula el parámetro velocidad para cada píxel.

$$
V=\left(d^{2 / 3} * S^{1 / 2}\right) / n
$$

\section{RESULTADOS}

Con la finalidad de validar la metodología planteada en este estudio y que pretende obtener la velocidad «estimada» (Ve) del flujo del agua en cauces efímeros y no aforados, se procedió a contrastar este valor con los datos de velocidad del flujo del agua medidos en campo (Vc). En la tabla 2 se presenta un resumen de los datos medidos en campo y los calculados a partir de los datos LiDAR 
Tabla 3

RESUMEN POR PARCELA DEL RADIO HIDRÁULICO (RHMED) EN M²/M, LA VARIANZA(V), LA PENDIENTE DE LA LÍNEA DEAGUA (S) EXPRESADA EN M/M, LA VELOCIDAD CALCULADAA PARTIR DE LOS DATOS DE CAMPO (VC) EN M/S, LA VELOCIDAD ESTIMADA (VE) EN M/S, LA N OBTENIDAA PARTIR DE LOS DATOS DE CAMPO (N_CAMPO) Y LAN $\operatorname{DEDUCIDA}\left(N \_D\right)$

\begin{tabular}{|c|c|c|c|c|c|c|c|}
\hline PARC & RHMed & V & $\mathbf{S}$ & Vc & Ve & n_Campo & n_D \\
\hline 1 & 0,169 & 0,003 & 0,002 & 0,498 & 0,332 & 0,028 & 0,041 \\
\hline 2 & 0,152 & 0,002 & 0,002 & 0,572 & 0,340 & 0,024 & 0,041 \\
\hline 3 & 0,208 & 0,003 & 0,003 & 0,379 & 0,436 & 0,048 & 0,042 \\
\hline 4 & 0,204 & 0,003 & 0,003 & 0,459 & 0,450 & 0,041 & 0,042 \\
\hline 5 & 0,267 & 0,003 & 0,001 & 0,239 & 0,222 & 0,040 & 0,043 \\
\hline 6 & 0,242 & 0,004 & 0,001 & 0,224 & 0,209 & 0,041 & 0,044 \\
\hline 7 & 0,162 & 0,004 & 0,011 & 0,712 & 0,735 & 0,044 & 0,043 \\
\hline 8 & 0,255 & 0,005 & 0,001 & 0,426 & 0,296 & 0,031 & 0,045 \\
\hline 9 & 0,131 & 0,012 & 0,015 & 0,626 & 0,598 & 0,050 & 0,053 \\
\hline 10 & 0,119 & 0,001 & 0,006 & 0,442 & 0,463 & 0,042 & 0,040 \\
\hline 11 & 0,153 & 0,002 & 0,007 & 0,475 & 0,574 & 0,049 & 0,040 \\
\hline 12 & 0,369 & 0,012 & 0,002 & 0,579 & 0,418 & 0,038 & 0,052 \\
\hline 13 & 0,153 & 0,004 & 0,012 & 0,748 & 0,720 & 0,041 & 0,043 \\
\hline 14 & 0,290 & 0,007 & 0,001 & 0,192 & 0,255 & 0,061 & 0,046 \\
\hline 15 & 0,258 & 0,006 & 0,000 & 0,169 & 0,143 & 0,039 & 0,046 \\
\hline 16 & 0,120 & 0,002 & 0,003 & 0,388 & 0,304 & 0,032 & 0,040 \\
\hline 17 & 0,045 & 0,003 & 0,008 & 0,571 & 0,263 & 0,019 & 0,042 \\
\hline 18 & 0,200 & 0,002 & 0,005 & 0,787 & 0,597 & 0,031 & 0,041 \\
\hline 19 & 0,098 & 0,002 & 0,013 & 0,436 & 0,589 & 0,054 & 0,040 \\
\hline 20 & 0,050 & 0,002 & 0,015 & 0,580 & 0,408 & 0,029 & 0,041 \\
\hline 21 & 0,170 & 0,002 & 0,028 & 1,268 & 1,229 & 0,040 & 0,041 \\
\hline 22 & 0,128 & 0,002 & 0,026 & 1,316 & 0,996 & 0,031 & 0,041 \\
\hline 23 & 0,231 & 0,004 & 0,014 & 1,414 & 1,025 & 0,031 & 0,043 \\
\hline 24 & 0,187 & 0,015 & 0,026 & 0,918 & 0,957 & 0,058 & 0,055 \\
\hline 25 & 0,262 & 0,006 & 0,012 & 0,916 & 1,003 & 0,050 & 0,046 \\
\hline 26 & 0,255 & 0,001 & 0,005 & 0,440 & 0,691 & 0,062 & 0,040 \\
\hline 27 & 0,229 & 0,023 & 0,011 & 1,059 & 0,617 & 0,038 & 0,065 \\
\hline 28 & 0,284 & 0,008 & 0,005 & 1,041 & 0,628 & 0,029 & 0,048 \\
\hline 29 & 0,125 & 0,036 & 0,003 & 0,185 & 0,169 & 0,071 & 0,078 \\
\hline 30 & 0,102 & 0,005 & 0,005 & 0,277 & 0,352 & 0,056 & 0,044 \\
\hline 31 & 0,151 & 0,046 & 0,013 & 0,364 & 0,375 & 0,089 & 0,087 \\
\hline 32 & 0,198 & 0,071 & 0,002 & 0,156 & 0,145 & 0,100 & 0,108 \\
\hline
\end{tabular}




\begin{tabular}{|c|c|c|c|c|c|c|c|}
\hline PARC & RHMed & $\mathbf{V}$ & $\mathbf{S}$ & $\mathbf{V c}$ & $\mathbf{V e}$ & n_Campo & n_D \\
\hline 33 & 0,124 & 0,001 & 0,001 & 0,285 & 0,235 & 0,033 & 0,040 \\
\hline 34 & 0,075 & 0,001 & 0,009 & 0,514 & 0,419 & 0,032 & 0,039 \\
\hline 35 & 0,114 & 0,006 & 0,007 & 0,336 & 0,449 & 0,061 & 0,045 \\
\hline 36 & 0,252 & 0,036 & 0,019 & 0,503 & 0,716 & 0,110 & 0,077 \\
\hline 37 & 0,254 & 0,010 & 0,009 & 0,514 & 0,780 & 0,076 & 0,050 \\
\hline 38 & 0,116 & 0,002 & 0,004 & 0,132 & 0,348 & 0,107 & 0,041 \\
\hline 39 & 0,215 & 0,013 & 0,022 & 0,949 & 0,975 & 0,056 & 0,054 \\
\hline 40 & 0,179 & 0,013 & 0,025 & 0,925 & 0,938 & 0,054 & 0,054 \\
\hline 41 & 0,410 & 0,105 & 0,003 & 0,241 & 0,243 & 0,130 & 0,128 \\
\hline 42 & 0,246 & 0,008 & 0,003 & 0,564 & 0,411 & 0,035 & 0,048 \\
\hline 43 & 0,299 & 0,017 & 0,003 & 0,405 & 0,445 & 0,063 & 0,058 \\
\hline
\end{tabular}

\section{Validación de la velocidad estimada (Ve)}

Los parámetros de entrada usados para estimar la velocidad (Ve) fueron: el radio hidráulico obtenido automáticamente (RhMed) a partir del MDE-LiDAR, la pendiente del agua medida en campo con GPS-RTK $(S)$ y la n de campo deducida (n_D) a partir del MDELiDAR.

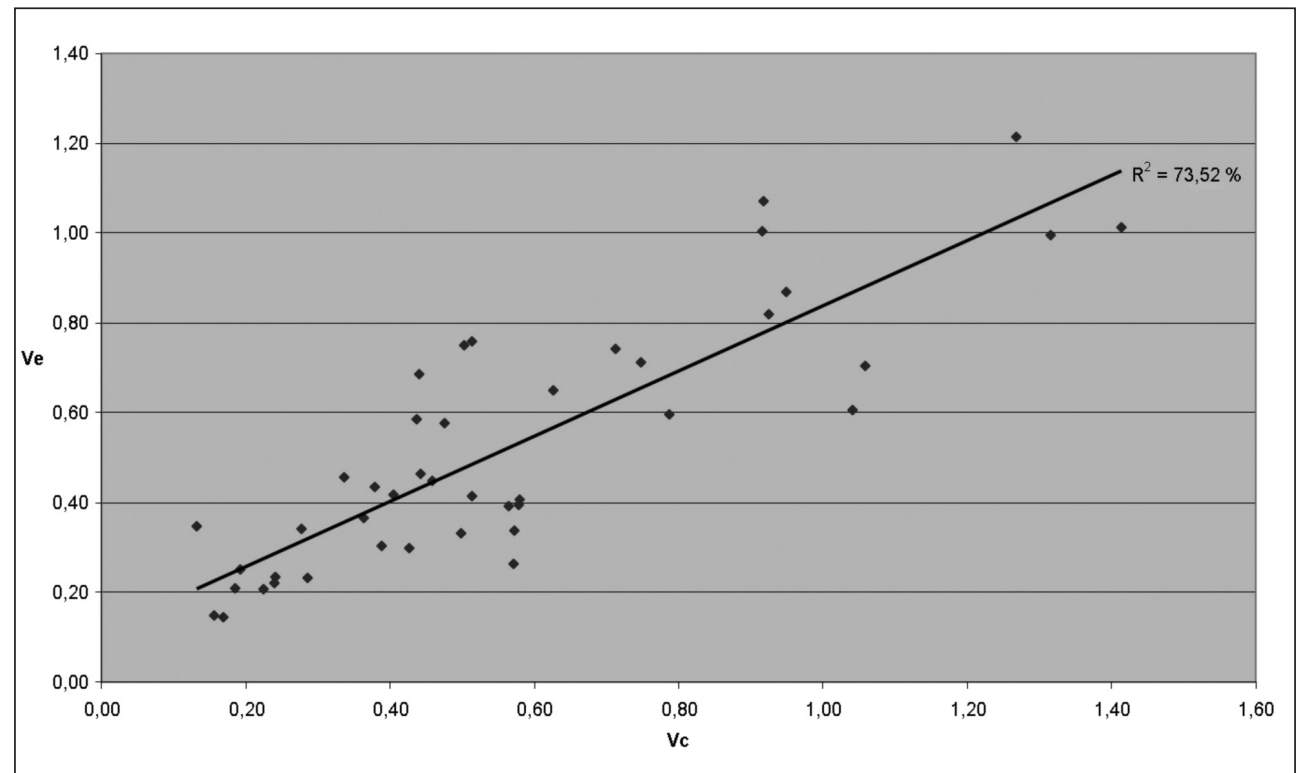


En la figura 6 se presenta el gráfico que relaciona la velocidad estimada (Ve) con la velocidad medida en campo (Vc). Como se observa, la variabilidad de la velocidad medida en campo es explicada en un 73,52\% por la velocidad estimada. Este resultado sugiere que la metodología propuesta para calcular la velocidad del flujo del agua a partir de datos LiDAR presenta un elevado potencial para obtener indirectamente la velocidad del flujo del agua en cauces naturales y la misma podría ser usada para generar mapas de velocidades de manera rápida, precisa, de áreas extensas y de difícil acceso.

\section{Elaboración de una primera aproximación a un mapa de velocidades}

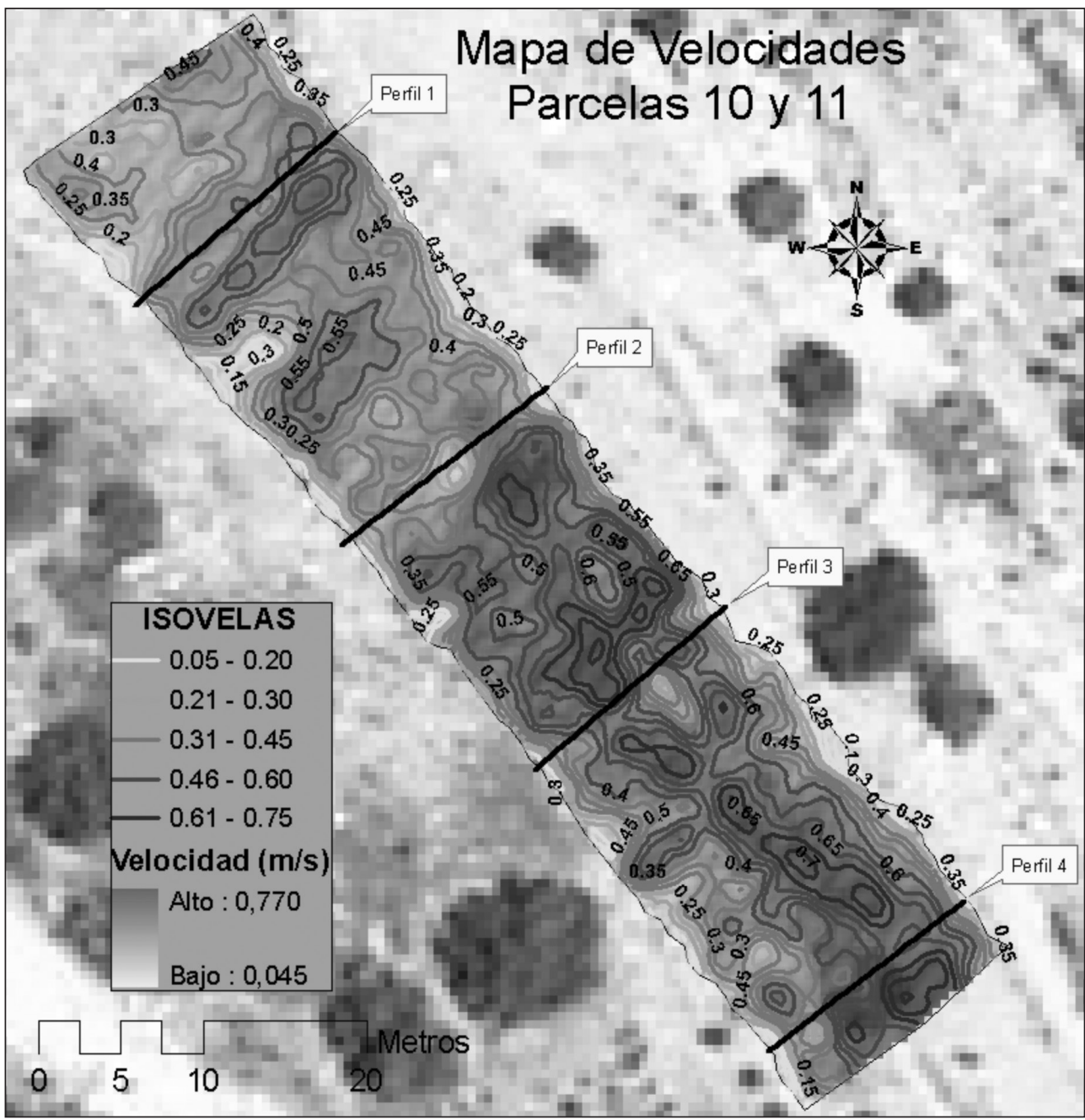


En la figura 7 se presenta una primera aproximación a lo que sería un mapa de velocidades de las parcelas 10 y 11. Se puede observar que las «isovelas» correspondientes a los valores de menor velocidad están ubicadas en las orillas del cauce en las parcelas 10 y 11 , por lo que es de esperar que las máximas velocidades se presenten en las zonas más profundas, precisamente donde hay menor rugosidad. Para construir este mapa se usó la fórmula de Manning - Limerinos [6]. Para obtener la profundidad (d) por píxel se construyó un nuevo modelo digital de elevaciones en la que la altitud dentro del cauce se correspondía con la cota del agua que se había medido con el GPS. Al restar este MDE «con agua» al MDE-LiDAR original se pudo obtener la profundidad de cada píxel. La pendiente $(S)$ era la misma para todos los píxeles dentro de una misma parcela y ésta se obtuvo a partir de las mediciones realizadas con el GPS y aplicando la ecuación [2]. La $n$ de Manning se obtuvo aplicando la ecuación [5] sobre cada píxel tomando como dato de entrada la varianza. Una vez que se disponen todos los términos se puede calcular directamente la ecuación [6], obteniendo el valor de velocidad para cada píxel.

Figura 8

GRAFICO DE LOS PERFILES TRANSVERSALES (a) Y EL DE LOS PERFILES DE VELOCIDADES (b). LA UBICACIÓN DE ESTOS PERFILES QUEDA REFLEJADA EN LA FIGURA 7

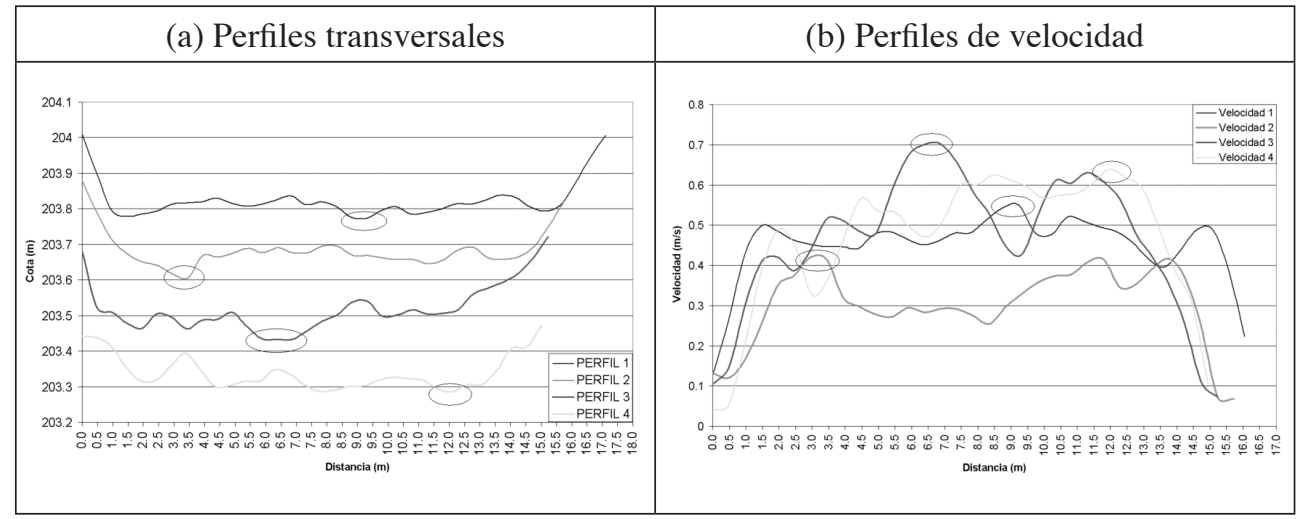

Con la finalidad de verificar la hipótesis de que los valores de mayor velocidad se corresponden con las zonas más profundas del cauce se construyeron 4 perfiles transversales (Fig. 7), de los cuales se recopiló la información necesaria para su confección (Fig. 8a). De igual forma se procedió para construir los perfiles de velocidad (Fig. 8b), confirmándose la hipótesis anterior al constatar, por ejemplo, que el punto más bajo del perfil transversal 3 , ubicado aproximadamente a $6,5 \mathrm{~m}$ del eje $\mathrm{x}$, se corresponde al valor de máxima velocidad en el perfil de velocidad 3. Lo mismo sucede en el perfil 4 a los $12 \mathrm{~m}$ aproximadamente. Por el contrario, en los valores de menor profundidad las velocidades siempre disminuyen. Resulta interesante que las mayores velocidades tienen lugar hacia la parte sur de las parcelas (Perfiles 3 y 4), este hecho podría estar relacionado directamente con la forma del cauce, ya que se va estrechando y profundizando hacia esta zona. 


\section{DISCUSIÓN}

Los estudios recientes realizados han demostrado el enorme potencial de estos datos para el cálculo de diversas variables de los cauces y de los llanos de inundación difíciles de obtener de forma masiva en el campo. Sin embargo, la rugosidad de los llanos de inundación obtenida a partir de datos LiDAR se ha mostrado como un parámetro dependiente de la escala de la topografía (Casas et al. 2010), lo que plantea una gran complejidad a la hora de utilizar estos datos en modelos hidráulicos. Por su parte, en este trabajo se ha evidenciado lo delicado que llega a ser el cálculo de la pendiente de la lámina de agua $(S)$, ya que sus variaciones son mínimas, alcanzando valores de sólo $0,0002 \mathrm{~m} / \mathrm{m}$ (valores medidos directamente en campo). La pendiente media (calculada promediando los valores obtenidos en ambos márgenes) es válida para medidas GPS-RTK, ya que sus precisiones son del orden de los $3 \mathrm{~cm}$ en altimetría, pero no así para los datos LiDAR, cuyas precisiones en altimetría llegan a ser de hasta $15 \mathrm{~cm}$. Al tratar de calcular la pendiente a partir de los datos LiDAR, se han deducido contrapendientes que no son coherentes con el flujo -lento pero real-que se observó en el campo. Esta situación es posible que se pueda minimizar haciendo las parcelas más grandes -por ejemplo de $100 \mathrm{~m}$ de longitud-, teniendo en cuenta que los ensayos realizados con parcelas de estas dimensiones han generado valores de pendiente de la lámina de agua $(S)$ coherentes. Sin embargo, no han sido introducidos en este estudio, debido a que los resultados obtenidos aún no han sido contrastados con datos tomados en campo con GPS. Esta cuestión queda abierta para futuros estudios de forma que, si la validación es satisfactoria, permitirán obtener la velocidad del flujo de forma rápida para zonas más amplias.

Otro aspecto de gran interés es la definición de la anchura del canal activo. Aunque existen diferentes maneras de obtenerlo, se han realizado algunas aproximaciones usando los datos de intensidad del primer pulso de puntos LiDAR tomados en un momento en el que circulaba agua por el cauce, obteniéndose resultados aceptables. Otra posibilidad sería usar ortofotos o imágenes de satélite de alta resolución tomadas con circulación hídrica en el cauce, aunque lamentablemente en este caso no se disponía de dicha información. Este parámetro es crucial a la hora de calcular el radio hidráulico $(R h)$ y la pendiente de la línea de agua $(S)$ de forma automatizada, ya que son dos variables muy sensibles a los pequeños cambios en la forma del cauce, tal y como se ha mencionado.

Por lo que respecta a la medición de la velocidad en cauces no aforados mediante la fórmula de Manning presenta algunos inconvenientes. La técnica de los flotadores esféricos utilizada en este artículo calcula la velocidad superficial y no la velocidad media de la corriente. Sin embargo, dada la escasa profundidad de los cauces medidos (menos de 0,2 m en la mayoría de los casos, Tabla 3) se minimiza el error generado haciéndolo asumible para los fines que se pretenden. Por otra parte, para condiciones de corrientes efímeras de régimen subcrítico y escaso calado, el método propuesto puede proporcionar buenos resultados. Sin embargo, su aplicación para el cálculo de la velocidad en grandes avenidas -sobre todo flash floods - es bastante discutible, dada la necesidad de utilizar otros métodos más apropiados para estimar la Vc y la dificultad de determinar con precisión la pendiente de la lámina de agua a partir de datos LiDAR y GPS-RTK, especialmente en situación de régimen turbulento supercrítico. Entendemos, en todo caso, que la línea de trabajo propuesto abre la posibilidad 
de elaborar mapas de isovelas siempre y cuando se pueda contar con modelos robustos realizados a partir de mediciones precisas de la velocidad del flujo.

Es de esperar, por tanto, que en un futuro no muy lejano se pueda obtener la velocidad del flujo del agua a partir de datos LiDAR, con lo que se disminuiría drásticamente el trabajo a realizar en campo, se podría obtener en zonas de difícil acceso de forma rápida y eficiente, automatizándose así todo el proceso.

\section{CONCLUSIONES}

Se ha propuesto, aplicado y validado una metodología semi-automatizada que tiene como finalidad obtener la velocidad promedio del flujo del agua en cauces naturales, no aforados, a partir de datos LiDAR y de mediciones realizadas en campo con GPS-RTK para obtener la pendiente de la lámina de agua y la geometría del cauce (parcela de estudio). Los resultados alcanzados sugieren que esta metodología presenta un alto potencial de aplicación para obtener la velocidad del flujo del agua, principalmente en situaciones donde se desee conocer este valor o por lo menos una primera aproximación rápida del mismo, en donde sea complicada su obtención aplicando las formas tradicionalmente usadas (por ejemplo zonas de difícil acceso en campo, cauces efímeros), o donde se pretenda conocer el valor de esta variable en una zona muy amplia. Gracias a la gran extensión de terreno que abarcan los datos LiDAR, se podrá obtener la velocidad del flujo del agua de forma masiva y rápida de una relativa extensa superficie del terreno y cuyas únicas limitaciones a la hora de calcularla serán la extensión de los datos LiDAR y del cauce cartografiado. Falta aún resolver adecuadamente el cálculo de la pendiente longitudinal de la lámina de agua, ya que aunque la podemos obtener de forma automática, faltaría contrastarla con mediciones de la misma en campo, quedando este aspecto abierto para su resolución en futuros estudios. También se abre la posibilidad de realizar mapas 3D que permitan cartografiar la variabilidad espacial de la velocidad del flujo del agua. También en un futuro se deberán contrastar los resultados obtenidos mediante la aplicación de modelos hidráulicos.

\section{AGRADECIMIENTOS}

Agradecemos la financiación por parte del Ministerio de Ciencia e Innovación y el FEDER, en el marco de los proyectos, CGL2009-14220-C02-02, CGL2009-14220-C02-01 y CGL2010-19591.

Agradecemos, por último, el apoyo y las observaciones realizadas por Pascual Garrigues, Luis García-Asenjo y Sergio Navarro del Departamento de Ingeniería Cartográfica, Geodesia y Fotogrametría de la Universitat Politècnica de València en el manejo de los sistemas GPS-RTK en modo VRS y de dos revisores anónimos cuyas correcciones han mejorado notablemente este trabajo.

\section{BIBLIOGRAFÍA}

ÁlVAREZ, FJ., NAVARRETE, LJ., RUIZ, C. y VICO, J. (2002): «Integración de la información relativa a la caracterización topográfica de la comarca olivarera "Sierra Magina" 
(Jaén) en un SIG vectorial» en XIV Congreso Internacional de Ingeniería Gráfica. Santander - España, Junio 2002.

BODANSKY, E., GRIBOV, A. y PILOUK, M. (2002): «Smoothing and Compression of Lines Obtained by Raster-to-Vector Conversion» en LNCS (Blostein, D. y Kwon, Y. B.) Berlin, Edit. Springer, 256-265.

CASAS, A.; LANE, S. N.; YU, D. and BENITO, G. (2010): «A method for parameterising roughness and topographic sub-grid scale effects in hydraulic modelling from LiDAR data». Hydrol. Earth Syst. Sci. Discuss., 7, 2261-2299.

CAVALLI, M., TAROLLI, P., MARCHI, L. y FONTANA, GD. (2008): «The effectiveness of Airborne LiDAR data in the recognition of channel-bed morphology». Catena, $\mathrm{n}^{\circ} 73$ (2008): 249-260. DOI: 10.1016/j.catena.2007.11.001.

CHOW, V., MAIDMENT, DR. y MAYS, LW. (1994): Hidrología aplicada. Buenos Aires. MCGraw Hill.

COLMENÁREZ, G., PARDO PASCUAL, JE., RUIZ, LA. y SEGURA-BELTRÁN, F. (2010): «Estudio de la Relación de la Rugosidad Topográfica obtenida a partir de datos LiDAR y GPS con el Coeficiente de Rugosidad n de Manning». Cuaternario y Geomorfología, $\mathrm{n}^{\mathrm{o}} 24$ (1-2), 135-151.

DOUGLAS, D. y PEUCKER, T. (1973): «Algorithms for the Reduction of the Number of Points Required to Represent a Digitized Line or its Caricature». The Canadian Cartographer, Vol. 10, $\mathrm{n}^{\circ}$ 2, Diciembre 1973, 112-122.

FRANKEL, KL., DOLAN, JF., FINKEL, RC., OWEN, LA. y HOEFT, JS. (2007): «Spatial variations in slip rate along the Death Valley-Fish Lake Valley fault system determined from LiDAR topographic data and cosmogenic ${ }^{10} \mathrm{Be}$ geochronology». Geophys. Res., $\mathrm{n}^{\mathrm{o}}$ 34: L18303. DOI: 10.1029/2007GL030549.

GILVEART, D., DAVIDS, C. y TYLER A. (2004): «The use of remotely sensed data to detect channel hidromorphology; river Tummel, Scotland». River Research and Applications, $\mathrm{n}^{\circ}$ 20, 795-811. DOI: 10.1002/rra.792.

GIOGIA, G. y BOMBARDELLI, FA. (2002): «Scaling and Similitary in Rough Channel Flows». Physical Review Letters, ${ }^{\circ}$ 88, 014501-1 - 014501-4. DOI:10.1103/PhysRevLett.88.014501.

GLENN, N., STREUTKER, D., CHADWICK, D., THACKRAY, G. y DORSCH S. (2006): «Analysis of LiDAR-derived topographic information for characterizing and differentiating landslide morphology and activity». Geomorphology, $\mathrm{n}^{\circ}$ 73, 131-148. DOI: 10.1016/j.geomorph.2005.07.006.

KATUL, G. G.; WIBERG, G. G.; ALBERSTON, J. and HORNBERGER, G. (2002): A mixing layer theory for flowresistance in shallow streams, Water Resour. Res., 38(11), 1250-1250.

KNIGHTON, AD. (1998): Fluvial Forms \& Processes: a new Perspective. Londres. Arnold.

KRAUS, K., and N. PFEIFER. (1998). Determination of terrain models in wooded areas with airborne laser scanner data. ISPRS Journal of Photogrammetry and Remote Sensing. 53: 193-203.

LOU, L. y CHEN, Y. (2008): «The Precision and Accuracy of Shangai VRS Network» en Observing our Changing Earth. (Sideris, M.G.). Perugia - Italy. Edit. International Association of Geodesy Symposia, 133: 719-723. 
McGAUGHEY, R. J. (2009). FUSION/LDV: Software for LIDAR data analysis and visualization: USDA Forest Service, Pacific Northwest Research Station.

MCKEAN, J. y ROERING, J. (2004): «Objective landslide detection and surface morphology mapping using high-resolution airborne laser altimetry». Geomorphology, $\mathrm{n}^{\circ} 57$, 331-351. DOI: 10.1016/S0169-555X(03)00164-8.

MOUTON, A. (2005): «Generating Stream Maps Using LiDAR Derived Digital Elevation Models and 10-m USGS DEM»Master of Science Thesis, University of Washington, 2005. Disponible en http://www.ruraltech.org/pubs/theses/mouton/mouton_ms_thesis. pdf.

ROBERT, A. (2003): River Processes: an Introduction to Fluvial Dynamics. Londres. Arnold.

STALEY, DM., WASKLEWICZ, TA. y BLASZCZYNSKI, JS. (2006): «Superficial patterns of debris flow deposition on alluvial fans in Death Valley, CA using airborne laser swath mapping data». Geomophology, n 74, 152-163. DOI: 10.1016/j.geomorph.2005.07.014.

WHITING, PJ. (2003): «Flow Measurement and Characterization» en Tools in Fluvial Geomorphology (GM Kondolf, H Piegay). John Wiley \& Sons Ltd: Chichester; 323-346. 
